$\underset{\text { clinical }}{\text { nephron }}$

Practice
Nephron 2018;139:120-130

DOI: 10.1159/000487093
Received: October 30, 2017

Accepted after revision: January 22, 2018 Published online: February 13, 2018

\title{
Assessing Extracellular Volume in Hemodialysis Patients Using Intradialytic Blood Pressure Slopes
}

\author{
Hao Liu ${ }^{a}$ Rong Lu ${ }^{b}$ Shani Shastria ${ }^{\text {Mark Sonderman }}{ }^{a}$ \\ Peter Noel Van Buren ${ }^{a, c}$ \\ a University of Texas Southwestern Medical Center, Department of Internal Medicine, Division of Nephrology, \\ Dallas, TX, USA; b University of Texas Southwestern Medical Center, Department of Clinical Sciences, Dallas, TX, USA; \\ 'Division of Nephrology, Medical Service, Veterans Affairs North Texas Health Care System, Dallas, TX, USA
}

\section{Keywords}

Hemodialysis · Bioimpedance spectroscopy · Intradialytic blood pressure slopes $\cdot$ Extracellular volume $\cdot$ Hypertension

\begin{abstract}
Background/Aims: Extracellular volume (ECV) overload is a mortality risk factor in hemodialysis patients, but no standard approach exists to objectively assess this clinically. We aimed to quantify relationships between slopes of repeated intradialytic blood pressure (BP) measurements and ECV. Methods: In a cross-sectional study of 71 hemodialysis patients, we calculated BP slopes from all intradialytic measurements using Gaussian regression. We measured extracellular and total body water (TBW) with bioimpedance spectroscopy. We analyzed unconditional and conditional associations between BP slope and volume metrics with mixed linear models and sensitivity analyses using non-linear intradialytic BP trajectory. Results: Mean systolic intradialytic BP slope (IBPS) was $-0.06(0.1) \mathrm{mm} \mathrm{Hg} / \mathrm{min}$. Post-dialysis extracellular water $(\mathrm{ECW}) /$ weight was the volume metric mostly strongly associated with slope $(r=0.34, p=0.007$ for unconditional analysis; $\beta=1.45, p=0.001$ for conditional analysis). Among subjects with post-dialysis systolic $B P \geq 130 \mathrm{~mm} \mathrm{Hg}$, the as-
\end{abstract}

\section{KARGER}

(C) 2018 S. Karger AG, Basel

E-Mail karger@karger.com

www.karger.com/nef sociation strengthened $(r=0.40, p=0.006 ; \beta=1.42, p=$ 0.003). ECV was more strongly associated with the BP slope than with pre-dialysis, post-dialysis, or delta systolic BP $(r=$ $-0.07,0.19,0.28 ; p=0.6,0.1,0.03$ ). In nonlinear models, BP trajectory also had the strongest association with post-dialysis ECW/body weight $(p<0.001)$. Conclusions: In hypertensive hemodialysis patients, measurements of ECV excess are more strongly associated with IBPSs than with pre-dialysis, post-dialysis, or change in systolic BP. Among varying volume metrics, post-dialysis ECW/weight has the strongest association with these slopes. Determining IBPS is a novel method to optimize clinical assessment of ECV in hemodialysis patients.

(c) 2018 S. Karger AG, Basel

\section{Introduction}

Extracellular volume (ECV) overload is strongly associated with increased mortality in end-stage renal disease patients $[1,2]$. Physical examination is unreliable for diagnosing ECV overload in hemodialysis patients [3], and there is no standard metric to define ECV overload in these patients in routine clinical practice. Bioimpedance 
spectroscopy (BIS) is a validated research tool for measuring fluid volumes in healthy individuals [4] and hemodialysis patients [5], and it is superior at identifying ECV excess compared to other noninvasive methods [6]. However, BIS has multiple limitations including contraindications in certain patients (e.g., those with defibrillators or pacemakers), inaccuracy in other patients (e.g., those with amputations or implanted metal), and limited availability that make it difficult to implement in routine clinical practice. A more readily implemented method to help assess ECV in the hemodialysis unit is necessary.

Intradialytic blood pressure $(\mathrm{BP})$ patterns have recently been linked to the degree of ECV overload $[7,8]$ with patients exhibiting intradialytic hypertension having higher ratios of ECV/TBW compared to patients with BP decreases. These findings require deeper investigation because (1) only $15 \%$ of hemodialysis patients regularly experience intradialytic hypertension $[9,10]$ and $(2)$ it is uncertain if this relationship extends across a continuous spectrum of intradialytic BP changes. Furthermore, the analysis of numerous BP measurements obtained all throughout dialysis may provide more information about ECV than can be obtained from just the pre-hemodialysis and/or post-hemodialysis measurements.

In this study, we aimed to determine the quantitative association of repeated intradialytic BP measurements and objective measurements of ECV using BIS in a heterogeneous group of hypertensive hemodialysis patients. We hypothesized that there would be a significant association between the intradialytic BP slope (IBPS) and post-dialysis extracellular water (ECW)/body weight. We also hypothesized that the post-HD ECW/body weight would have a stronger association with the IBPS than with pre, post, or delta systolic BP.

\section{Methods}

\section{Design and Subjects}

This study combined subjects from a previously conducted case-control study with additional consecutively enrolled hypertensive hemodialysis patients. The original case-control study compared measurements of BIS and noninvasive cardiac output monitoring in patients with intradialytic hypertension and other hypertensive hemodialysis controls [8]. Inclusion criteria were age $>18$ years, hemodialysis vintage $>1$ month, and hypertension defined by Kidney Disease Outcomes Quality Initiative (KDOQI) guidelines (systolic BP $>140 \mathrm{~mm} \mathrm{Hg}$ pre-dialysis or $>130 \mathrm{~mm} \mathrm{Hg}$ post-dialysis) [11]. The case subjects for the original study had intradialytic hypertension defined by systolic BP increase $>10 \mathrm{~mm}$ $\mathrm{Hg}$ from pre- to post-dialysis in $4 / 6$ screening treatments. The controls in the original study had systolic BP decreases $>10 \mathrm{~mm} \mathrm{Hg}$ from pre- to post dialysis in 4/6 screening treatments. Exclusion criteria were major extremity amputation; cardiac defibrillator, pacemaker or coronary artery stent; pregnancy, metal prosthesis, or failure to achieve dry weight consistently. We then consecutively enrolled an additional 35 hypertensive hemodialysis patients meeting the above general inclusion and exclusion criteria with no requirement of any specific intradialytic BP pattern. Data from all 71 subjects were included in this study.

Subjects signed written informed consent before procedures. The University of Texas Southwestern Medical Center Institutional Review Board approved the protocol. All procedures were in accordance with the Declaration of Helsinki. The study was part of a registered clinical trial, NCT01862497 [12].

\section{Study Procedures}

Bioimpedance Spectroscopy

We used whole-body multifrequency BIS (Impedimed SFB7, Carlsbad CA, USA) to obtain ECW and total body water (TBW) measurements. Measurements were obtained before and $30 \mathrm{~min}$ after a mid-week hemodialysis treatment, while the patient was in the supine position with electrodes placed on the wrist, hand, foot, and ankle contralateral to the hemodialysis access. Intracellular water (ICW) was calculated by subtracting the ECW from the TBW. We also calculated fluid overload (L) before and after dialysis using an equation previously validated in other bioimpedance devices: $1.136^{*} \mathrm{ECW}-0.430^{*} \mathrm{ICW}-0.114^{*}$ weight $[13,14]$.

\section{Cardiovascular Hemodynamics}

We used impedance cardiography (Non Invasive Cardiac Output Monitor from Cheetah Medical Inc., Newton Center, MA, USA) to obtain various hemodynamic measurements. Electrodes were placed on the anterior and posterior of the thoracic cavity. Before and $30 \mathrm{~min}$ after the mid-week treatment, we obtained measurements of cardiac output, cardiac index (CI), and mean arterial BP from which total peripheral resistance index (TPRI) was automatically calculated. The device simultaneously measures mean transthoracic electrical impedance determined by the thoracic fluid content [15]. Thoracic fluid content is expressed as 1,000 times the reciprocal of measured impedance (1,000/impedance). Thoracic fluid content obtained from this device differentiates acute heart failure from noncardiac etiologies of dyspnea [16] and correlates strongly with fluid removal in hemodialysis patients [15].

Measurement of Endothelial Cell-Derived Vasomediators

Before and after a mid-week dialysis treatment, we collected and centrifuged $10 \mathrm{~mL}$ blood. Plasma was stored in a -80 degree Celsius freezer. We measured endothelin-1 (ET-1) with quantitative sandwich enzyme immunoassay technique with Human Endothelin-1 Immunoassay (Quantiglo) and asymmetric dimethylarginine (ADMA) by using competitive enzyme linked immunosorbent assay (Biovendor) with a microtiter plate format.

\section{Intradialytic BP Slopes}

BP measurements were obtained at least at 30 min intervals throughout the hemodialysis treatment using the sphygmomanometers connected to the hemodialysis machine. Measurements were electronically recorded in patients' medical records. By using every measurement recorded after the treatment started, we calculated IBPS ( $\mathrm{mm} \mathrm{Hg} / \mathrm{min}$ ) as detailed below. 
Statistical Analysis

Continuous measurements are summarized using sample mean with SD for normal sample distributions, and otherwise presented as median with interquartile range. We first estimated IBPS separately for each patient using univariate Gaussian Regression (with time variable included as a linear term). We then used Pearson correlation in univariate analysis to estimate the unconditional correlation between IBPS and the following volume metrics both before and after dialysis: ECW, TBW, the ratio of ECW/TBW, the ratio of ECW/body weight, fluid overload, and thoracic fluid content in our subjects. For this primary outcome, statistical significance was determined to be $p<0.05$. To quantify the conditional association between IBPS with these volume metrics, we then applied linear regression models (with IBPS as the response variable) to adjust for the confounding effects of gender, presence of diabetes, and the rate of ultrafiltration during dialysis $(\mathrm{mL} / \mathrm{kg} / \mathrm{h})$. Using similar models, we then quantified unconditional and conditional (adjusting for gender, diabetes, and ultrafiltration rate) associations between the post-dialysis ECW/body weight with other metrics of dialysis unit BP: pre-dialysis, post-dialysis, and delta (post-pre) systolic BP. In exploratory analyses, we used Pearson correlation and linear regression models to determine the unconditional and conditional associations between IBPS and post-HD ECW/body weight in subgroups (with or without diabetes, positive or negative IBPS). We also used mixed linear models to determine how additional individual variables proposed to influence BP (TPRI, CI, ET-1, ADMA, dialysate sodium, dialysate calcium) were associated with IBPS and how they influenced the association between IBPS and post-HD ECW/body weight.

In a sensitivity analysis, we used mixed-effect models to access the population-level association between a nonlinear intradialytic BP trajectory with the ECV metrics to control possible correlation among BP measurements from the same patient. Since IBPS enforced a linear structure, we implemented the mixed-effect models to include all repeated measurements without the intermediate abstraction step of mapping each trajectory to an IBPS value. This analysis not only allows us to model $\mathrm{BP}$ as a nonlinear function of time but also serves as a sensitivity analysis to show the relative association strength estimated using IBPS is consistent with the estimates based on models without the linearity assumption. For unconditional association analyses, we used the mixed-effect model containing only the time variable as the base model. The unconditional association between BP trajectory and volume metrics was then quantified using the likelihood ratio of comparing the effect of adding this volume metric and an interaction term between this metric and time. For conditional analyses, we used the mixed-effect model containing gender, diabetes, and ultrafiltration rate in addition to time. The conditional association between IBP trajectory and a volume metric was quantified using the likelihood ratio of comparing the effect of adding this volume metric and an interaction term with time to this adjusted base model. All statistical analyses were performed with $\mathrm{R}$ version 3.3.2.

\section{Results}

\section{Subjects}

There were 71 subjects enrolled with a predominance of African-American and Hispanic subjects and 38\% fe-
Table 1. Patient characteristics $(n=71)$

Patient demographics

Age, years, $n(\%)$

Female, \%

$49.4(12)$

African American, \%

38

58

Hispanic, \%

Diabetic, \%

Estimated dry weight, $\mathrm{kg}, n(\%)$

30

55

$86.1(19)$

Dialysis prescription, $n$ (\%)

Dialysis time, min

234 (19)

Dialysis blood flow rate, $\mathrm{mL} / \mathrm{min}$

$415(92)$

$674(113)$

Dialysis dialysate flow rate, $\mathrm{mL} / \mathrm{min}$

$138(0.8)$

$2.12(0.3)$

$2.51(0.1)$

Dialysate calcium, $\mathrm{mmol} / \mathrm{L}$

Treatment characteristics, $n(\%)$

Pre-dialysis weight, $\mathrm{kg}$

Post-dialysis weight, $\mathrm{kg}$

$89.1(20)$

$86.4(20)$

Percentage of interdialytic weight gain to dry weight

Ultrafiltration rate, $\mathrm{mL} / \mathrm{kg} / \mathrm{h}$

$2.95(1.8)$

$8.07(3.8)$

Medication profile, \%

Angiotensin converting enzyme inhibitor

Angiotensin receptor blocker

34

Beta adrenergic receptor antagonist

Alpha adrenergic receptor antagonist

Calcium channel blocker

Hydralazine

Clonidine

Sensipar

Erythropoetin stimulating agent, dose

(\%, units/treatment)

Calcium containing phosphate binder

Non-calcium containing phosphate binder

Statin

Monthly lab values, $n$ (\%)

Blood urea nitrogen, $\mathrm{mmol} / \mathrm{L}$

Serum creatinine, $\mu \mathrm{mol} / \mathrm{L}$

$\mathrm{Kt} / \mathrm{V}$

Hemoglobin, g/L

Ferritin, pmol/Lng/mL

Iron saturation, \%

Parathyroid hormone, ng/LL

Serum calcium, $\mathrm{mmol} / \mathrm{L}$

Serum phosphorus, $\mathrm{mmol} / \mathrm{L}$

Serum albumin, $\mathrm{g} / \mathrm{L}$

PCR

34
20
76
58
62
21
13
30

$80,4,420(4,400)$

58

42

34

$19.9(5.7)$

$793(210)$

$1.48(0.3)$

$106(13)$

$1,320(740)$

$30.0(13)$

$647(720)$

$2.30(0.2)$

$1.98(0.7)$

$38.1(3)$

$1.07(0.4)$

male subjects. Of 262 subjects screened, 36 enrolled in the original case-control study (18 subjects per group) [8]. An additional 35 subjects were subsequently enrolled for this analysis. Detailed patient characteristics are listed in Table 1. During the mid-week hemodialysis treatment, the mean pre-HD, post-HD, and lowest systolic BP were 156 (19), 143 (22), and 118 (21) mm Hg respectively. The 
Table 2. Bioimpedance-based fluid measurements

$\begin{array}{lc}\text { Pre-dialysis TBW, L }(n=69) & 47.9(9.4) \\ \text { Post-dialysis TBW, L }(n=67) & 45.0(9.1) \\ \text { Pre-dialysis ECW, L }(n=69) & 22.2(4.1) \\ \text { Post-dialysis ECW, L }(n=67) & 20.0(3.9) \\ \text { Pre-dialysis ICW, L }(n=69) & 25.7(6.1) \\ \text { Post-dialysis ICW, L }(n=67) & 25.0(6.0) \\ \text { Pre-dialysis extracellular/TBW }(n=70) & 0.47(0.04) \\ \text { Post-dialysis extracellular/TBW }(n=65) & 0.45(0.04) \\ \text { Pre-dialysis ECW/body weight }(n=69) & 0.26(0.05) \\ \text { Post-dialysis ECW/body weight }(n=65) & 0.24(0.05) \\ \text { Delta ECW/body weight }(n=65) & -0.02(0.01) \\ \text { Pre-dialysis fluid overload, L }(n=69) & 4.07(3.5) \\ \text { Post-dialysis fluid overload, L }(n=67) & 2.15(3.3) \\ \text { Pre-dialysis thoracic fluid content }(n=59) & 44.4(11) \\ \text { Post-dialysis thoracic fluid content }(n=59) & 39.6(12)\end{array}$

ECW, extracellular water; TBW, total body water; ICW, intracellular water.

Table 3. Associations between bioimpedance-based volume measurements with intradialytic blood pressure slopes for all subjects in univariate analyses (Pearson correlation) and multivariate analyses (linear regression models) as well as univariate analyses in subjects with systolic blood pressure $>130 \mathrm{~mm} \mathrm{Hg}$ after dialysis

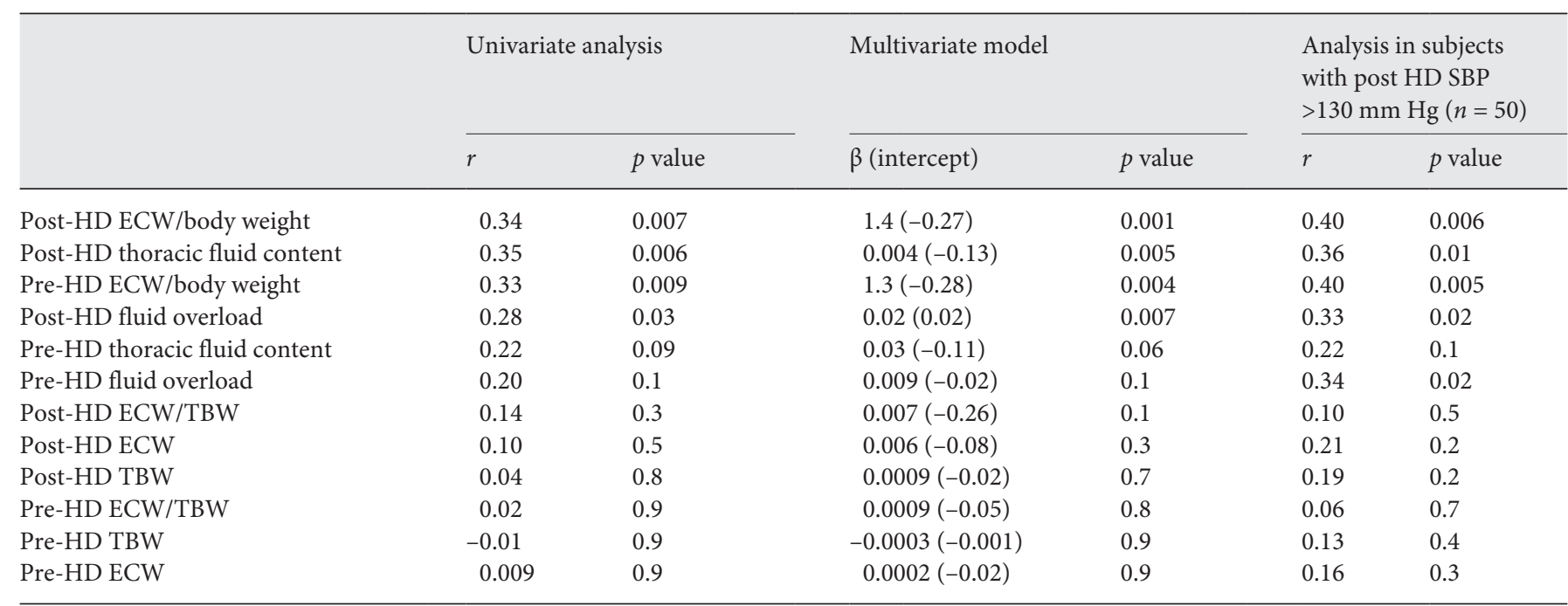

HD, hemodialysis; ECW, extracellular water; TBW, total body water. Multivariate model controlling for gender, presence of diabetes and ultrafiltration rate.

mean change in systolic BP from pre to post-HD was -12.7 (29) $\mathrm{mm} \mathrm{Hg}$. The mean number of intradialytic BP measurements per subject was 9.85 (1.5), and the mean IBPS (available for 65 subjects) was $-0.06(0.1) \mathrm{mm} \mathrm{Hg} /$ min.

\section{Associations between BIS and Intradialytic BP}

Table 2 shows pre- and post-HD BIS measurements. In univariate analyses, IBPS had significant correlations with pre-HD ECW/body weight $(r=0.33, p=0.009)$ and
post-HD measurements of ECW/body weight $(r=0.34$, $p=0.007)$, fluid overload $(r=0.28, p=0.03)$, and thoracic fluid content $(r=0.35, p=0.006)$. The remaining correlations are presented in Table 3. In the adjusted model, IBPS maintained significant associations with pre-HD ECW/body weight $(\beta=1.27, p=0.004)$ and postHD fluid overload ( $\beta=0.02, p=0.007)$ and thoracic fluid content $(\beta=0.004, p=0.005)$. The strongest association was again with post-HD ECW/body weight ( $\beta=1.45, p=$ 0.001 ; Table 3 ). In this model, the intercept was -0.27 . 


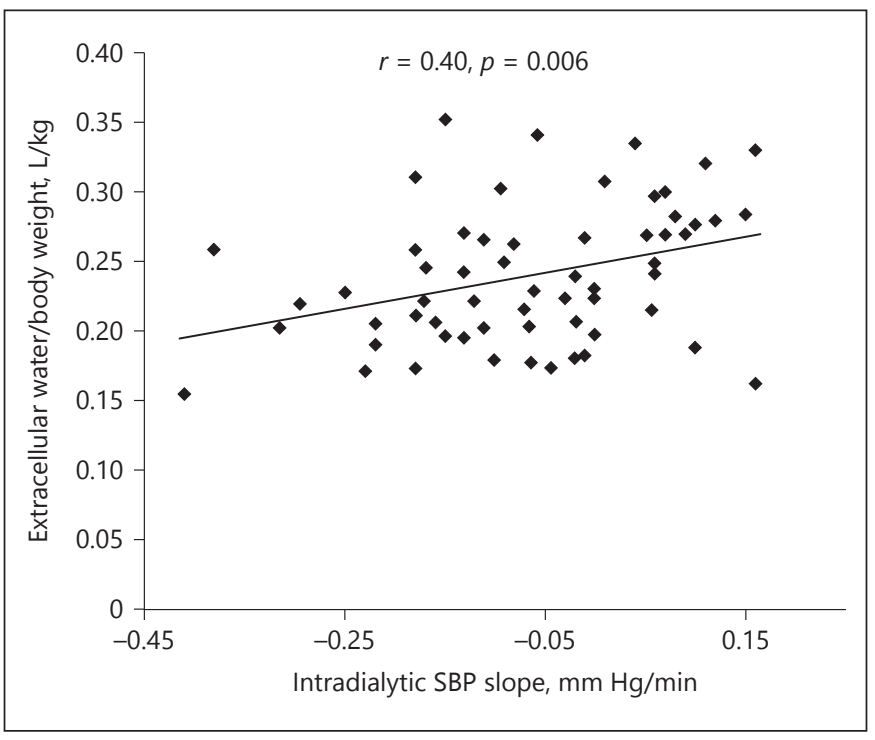

Fig. 1. The scatterplot of the ratio of extracellular water/body weight $(\mathrm{L} / \mathrm{kg})$ on the $\mathrm{y}$-axis and the intradialytic blood pressure slope $(\mathrm{mm} \mathrm{Hg} / \mathrm{min})$ on the $\mathrm{x}$-axis among subjects with post-hemodialysis systolic blood pressure (SBP) $>130 \mathrm{~mm} \mathrm{Hg}$. The correlation coefficient for these variables is $0.40(p=0.006)$.

Diabetes remained independently associated with the slope $(\beta=-0.08, p=0.03)$, but gender $(\beta=-0.05, p=0.2)$ and ultrafiltration rate $(\beta=-0.008, p=0.09)$ did not have an independent association. In all other conditional analyses for the other variables related to ECV, other than the post-HD fluid overload, the intercept for IBPS was negative. In all these analyses, diabetes had negative independent associations with IBPS with at least trends for statistical significance $(p<0.1$ in all models).

\section{Associations between ECW/Body Weight and Other}

\section{Peridialytic BP Metrics}

In unconditional analyses, the correlation coefficients for post-HD ECW/body weight with pre-HD, post-HD and delta systolic BP were $-0.07(p=0.6), 0.19(p=0.1)$, and $0.28(p=0.03)$ respectively. In models adjusting for gender, ultrafiltration rate and presence of diabetes, there were significant associations with post-HD $(\beta=0.0007$, $p=0.007)$ and delta systolic BP $(\beta=0.0006, p<0.001)$. However, the strongest association remained with IBPS $(\beta=0.13, p=0.001)$.

\section{Modeling in Subjects with and without}

Post-Hemodialysis Hypertension

Correlations between IBPS and different volume metrics were strengthened among 50 subjects with post-HD systolic $\mathrm{BP}>130 \mathrm{~mm} \mathrm{Hg}$ (Table 3). Again, post-HD ECW/ body weight had the strongest correlation $(r=0.40, p=$ 0.006; Fig. 1). The correlation for pre-HD ECW/body weight also increased to $0.40(p=0.005)$. Both pre- and post-HD ECW/body weight remained significantly associated with IBPS in these subjects in analyses adjusting for age, gender, and ultrafiltration rate $(\beta=1.3, p=0.005$ and $\beta=1.4, p=0.003$, respectively). Among subjects with post-HD systolic $\mathrm{BP}<130 \mathrm{~mm} \mathrm{Hg}(n=14)$, the correlations for pre- and post-hemodialysis ECW/body weight were $-0.35(p=0.2)$ and $-0.10(p=0.8)$.

\section{Sensitivity Analysis of Intradialytic BP Trajectory}

In a sensitivity analysis, a nonlinear model of intradialytic BP trajectory was analyzed using the same ECV metrics. In both unconditional and conditional models, ECW/body weight had the strongest association with intradialytic BP trajectory (Table 4). There were also significant associations with pre-hemodialysis ECW/body weight and pre and post-HD fluid overload and thoracic fluid content.

\section{Association between Cardiac Hemodynamics and IBPS}

The association between post-HD ECW/body weight and IBPS remained statistically significant when the following variables were individually added into multivariate linear regression analyses: dialysate sodium concentration, dialysate calcium concentration, intradialytic change in TPRI, intradialytic change in CI, and intradialytic change in ADMA (Table 5). It became one of marginal significance while controlling for intradialytic change in ET-1. The intradialytic change in TPRI was associated with IBPS in these models, but no other variables were. In a final model controlling for age, ultrafiltration rate and presence of diabetes, both post-HD ECW/body weight and change in TPRI continued to have significant associations with IBPS (Intercept -0.1 ; delta TPRI $\beta<0.0001, p=$ 0.002 ; post-HD ECW/body weight $\beta=0.8, p=0.03$ ).

\section{Subgroup Analyses of Patients with Negative and} Positive IBPSs

We assessed whether or not the strength of the correlation between ECV/weight and IBPS varied among subjects with more similar IBPS. There were 44 subjects with negative slopes (IBPS $<0 \mathrm{~mm} \mathrm{Hg}$ ) and 21 subjects with positive slopes (IBPS $\geq 0 \mathrm{~mm} \mathrm{Hg}$ ). Characteristics of subjects with positive and negative slopes are shown in Table 6 . The correlation coefficient for IBPS and ECW/body weight was $0.11(p=0.5)$ for subjects with 
Table 4. Associations of bioimpedance-based measurements with nonlinear intradialytic blood pressure trajectories in both univariate and multivariate models

\begin{tabular}{llllll}
\hline & \multicolumn{2}{l}{ Univariate model } & & & \multicolumn{2}{l}{ Multivariate model } \\
\cline { 2 - 3 } \cline { 5 - 6 } & likelihood ratio & $p$ value & & likelihood ratio & $p$ value \\
\hline Post-HD ECW/body weight & 39.7 & $<0.001$ & & 48.9 & $<0.001$ \\
Pre-HD ECW/body weight & 40.5 & $<0.001$ & & 48.7 & $<0.001$ \\
Post-HD thoracic fluid content & 36.4 & $<0.001$ & & 37.7 & $<0.001$ \\
Post-HD fluid overload & 24.9 & $<0.001$ & & 30.9 & $<0.001$ \\
Pre-HD thoracic fluid content & 23.3 & $<0.001$ & & 27.4 & $<0.001$ \\
Pre-HD fluid overload & 17.1 & $<0.001$ & & 22.8 & 0.001 \\
Post-HD ECW/TBW & 9.02 & 0.01 & & 9.51 & 0.009 \\
Pre-HD TBW & 3.43 & 0.2 & & 1.30 & 0.2 \\
Post-HD ECW & 3.26 & 0.2 & & 3.14 & 0.5 \\
Post-HD TBW & 3.17 & 0.2 & & 1.54 & 0.6 \\
Pre-HD ECW & 2.29 & 0.3 & & 0.99 & 0.5 \\
Pre-HD ECW/TBW & 1.02 & 0.6 & 1.57 & 0.5 \\
\hline
\end{tabular}

HD, hemodialysis; ECW, extracellular water; TBW, total body water. Multivariate model controls for gender, presence of diabetes, and ultrafiltration rate.

Table 5. Results of multivariate linear regression models where IBPS is the outcome variables and post-HD ECW/ body weight + listed variable below are the predictor variables

\begin{tabular}{llllll}
\hline Variable & Intercept & $\begin{array}{l}\beta \text { for listed } \\
\text { variable }\end{array}$ & $\begin{array}{l}p \text { value for } \\
\text { listed variable }\end{array}$ & $\begin{array}{l}\beta \text { for post-HD } \\
\text { ECW/body weight }\end{array}$ & $\begin{array}{l}p \text { value for } \\
\text { post-HD ECW/ } \\
\text { body weight }\end{array}$ \\
\hline Delta TPRI, dynes $/ \mathrm{s}^{2} \mathrm{~cm}^{2} / \mathrm{m}^{2}$ & -0.21 & $6.48 \mathrm{e}^{-5}$ & 0.0004 & 0.69 & 0.03 \\
Delta CI, $\mathrm{L} / \mathrm{min} / \mathrm{m}^{2}$ & -0.28 & -0.02 & 0.6 & 0.90 & 0.008 \\
Dialysate sodium, $\mathrm{mmol} / \mathrm{L}$ & -3.09 & 0.02 & 0.3 & 0.93 & 0.006 \\
Dialysate calcium, $\mathrm{mmol} / \mathrm{L}$ & -0.31 & 0.008 & 0.9 & 0.92 & 0.007 \\
Delta ET-1, $\mathrm{pg} / \mathrm{mL}$ & -0.23 & -0.05 & 0.1 & 0.7 & 0.06 \\
Delta ADMA, $\mu \mathrm{mol} / \mathrm{L}$ & -0.33 & -0.12 & 0.2 & 0.94 & 0.007 \\
\hline
\end{tabular}

IBPS, intradialytic blood pressure slope; HD, hemodialysis; ECW, extracellular water; TPRI, total peripheral resistance index; CI, cardiac index; ET-1, endothelin-1; ADMA, asymmetric dimethylarginine.

negative slopes and $0.08(p=0.8)$ for subjects with positive slopes. In our multivariable regression model controlling for age, ultrafiltration rate, and diabetes, the parameter estimate for post-HD ECW/body weight was 0.5 in those with negative slopes $(p=0.4)$ and 0.72 in those with positive slopes $(p=0.01)$. In these models, age and ultrafiltration rate had no significant effect on the IBPS; the presence of diabetes had a significant effect in those with negative slopes $(\beta=-0.08, p=0.02)$ but did not have a significant effect in those with positive slopes $(\beta=-0.02, p=0.3)$.

\section{Subgroup Analyses of Patient with and without Diabetes}

There were 37 subjects with confirmed history of diabetes, and there were 23 subjects with a confirmed history of not having diabetes. The IBPS were -0.09 $(0.1)$ in those with and $-0.03(0.1)$ in those without diabetes $(p=0.03)$. The univariate correlations between IBPS and post-HD ECW/body weight were 0.38 ( $p=$ $0.03)$ in those with and $0.42(p=0.04)$ in those without diabetes. 
Table 6. Comparison of subjects with negative and positive IBPSs

\begin{tabular}{|c|c|c|c|}
\hline & Negative slopes $(n=44)$ & Positive slopes $(n=21)$ & $p$ value \\
\hline Age, years & $50.5(11)$ & $47.1(14)$ & 0.3 \\
\hline Diabetes (\% with) & 66 & 53 & 0.1 \\
\hline Intradialytic blood pressure slope, $\mathrm{mm} \mathrm{Hg} / \mathrm{min}$ & $-0.13(0.1)$ & $0.08(0.05)$ & $<0.001$ \\
\hline Pre-HD SBP, mm Hg & $161(17)$ & $144(19)$ & 0.002 \\
\hline Post-HD SBP, mm Hg & $138(22)$ & $154(20)$ & 0.008 \\
\hline Delta SBP, mm Hg & $-22.7(27)$ & $9.25(24)$ & $<0.001$ \\
\hline SBP Nadir, mm Hg & $115(21)$ & $127(14)$ & 0.01 \\
\hline Pre-HD DBP, mm Hg & $91.5(13)$ & $89.2(24)$ & 0.7 \\
\hline Post-HD DBP, mm Hg & $81.8(11)$ & $92.9(18)$ & 0.01 \\
\hline Delta DBP, mm Hg & $-9.70(13)$ & $2.5(12)$ & $<0.001$ \\
\hline Pre-HD TPRI, dynes $/ \mathrm{s} / \mathrm{cm}^{2} / \mathrm{m}^{2}$ & $3,250(800)$ & $3,180(820)$ & 0.7 \\
\hline Post-HD TPRI, dynes $/ \mathrm{s} / \mathrm{cm}^{2} / \mathrm{m}^{2}$ & $2,760(610)$ & $3,400(870)$ & 0.006 \\
\hline Delta TPRI, dynes $/ \mathrm{s} / \mathrm{cm}^{2} / \mathrm{m}^{2}$ & $-486(747)$ & $183(920)$ & 0.008 \\
\hline Pre-HD CI, $\mathrm{L} / \mathrm{min} / \mathrm{m}^{2}$ & $2.98(0.6)$ & $2.83(0.5)$ & 0.3 \\
\hline Post-HD CI, L/min $/ \mathrm{m}^{2}$ & $3.09(0.5)$ & $2.85(0.6)$ & 0.1 \\
\hline Delta CI, $\mathrm{L} / \mathrm{min} / \mathrm{m}^{2}$ & $0.11(0.5)$ & $0.02(0.4)$ & 0.5 \\
\hline Pre-HD pulse, beats per minute & $78.1(9.3)$ & $72.8(14)$ & 0.1 \\
\hline Post-HD pulse, beats per minute & $81.2(11.9)$ & $77.6(12)$ & 0.3 \\
\hline Delta pulse, beats per minute & $3.11(7.8)$ & $4.03(8.8)$ & 0.7 \\
\hline Pre-HD ECW/body weight, L/kg & $0.25(0.05)$ & $0.28(0.05)$ & 0.008 \\
\hline Post-HD ECW/body weight, L/kg & $0.23(0.04)$ & $0.27(0.05)$ & 0.005 \\
\hline Delta ECW/body weight, L/kg & $-0.02(0.01)$ & $-0.01(0.02)$ & 0.1 \\
\hline Pre-HD TFC & $42.5(11)$ & $49.9(11)$ & 0.02 \\
\hline Post-HD TFC & $36.5(9.3)$ & $47.5(13)$ & 0.002 \\
\hline Delta TFC & $-5.94(4.6)$ & $-2.33(7.1)$ & 0.05 \\
\hline Pre-HD fluid overload, L & $3.55(3.5)$ & $5.41(3.3)$ & 0.04 \\
\hline Post-HD fluid overload, L & $1.5(3.0)$ & $3.74(3.5)$ & 0.02 \\
\hline Pre-HD ET-1, pg/mL & $1.75(0.5)$ & $3.00(2.0)$ & 0.02 \\
\hline Post-HD ET-1, pg/mL & $1.98(0.6)$ & $2.85(1.6)$ & 0.05 \\
\hline Delta ET-1, pg/mL & $0.22(0.5)$ & $-0.20(0.7)$ & 0.04 \\
\hline Pre-HD ADMA, $\mu \mathrm{mol} / \mathrm{L}$ & $0.78(0.2)$ & $0.75(0.2)$ & 0.5 \\
\hline Post-HD ADMA, $\mu \mathrm{mol} / \mathrm{L}$ & $0.51(0.2)$ & $0.40(0.1)$ & 0.01 \\
\hline Delta ADMA, $\mu \mathrm{mol} / \mathrm{L}$ & $-0.28(0.2)$ & $-0.34(0.1)$ & 0.2 \\
\hline Pre-HD weight, kg & $91.0(20)$ & $82.4(18)$ & 0.09 \\
\hline Post-HD weight, kg & $88.2(20)$ & $79.8(18)$ & 0.09 \\
\hline Estimated dry weight, kg & $87.9(20)$ & $79.7(17)$ & 0.1 \\
\hline Ultrafiltration rate, $\mathrm{mL} / \mathrm{kg} / \mathrm{h}$ & $8.06(3.8)$ & $8.31(4.0)$ & 0.8 \\
\hline Dialysate sodium, mmol/L & $138(0.8)$ & $138(0.9)$ & 0.8 \\
\hline Dialysate potassium, $\mathrm{mmol} / \mathrm{L}$ & $2.14(0.3)$ & $2.05(0.2)$ & 0.2 \\
\hline Dialysate calcium, mmol/L & $2.50(0.1)$ & $2.5(0)$ & 0.9 \\
\hline Blood urea nitrogen, $\mathrm{mmol} / \mathrm{L}$ & $20.3(4.6)$ & $18.1(6.1)$ & 0.2 \\
\hline Serum creatinine, $\mu \mathrm{mol} / \mathrm{L}$ & $770(180)$ & $824(220)$ & 0.4 \\
\hline Serum potassium, $\mathrm{mmol} / \mathrm{L}$ & $4.89(0.6)$ & $4.67(0.5)$ & 0.2 \\
\hline $\mathrm{Kt} / \mathrm{V}$ & $1.49(0.3)$ & $1.48(0.2)$ & 0.9 \\
\hline Hemoglobin, g/L & $107(12)$ & $103(15)$ & 0.4 \\
\hline Ferritin, pmol/L & $1,290(809)$ & $1,380(562)$ & 0.7 \\
\hline Iron saturation & $29.9(12)$ & $27.8(11)$ & 0.5 \\
\hline PTH, ng/L & $707(820)$ & $521(550)$ & 0.3 \\
\hline Serum calcium, mmol/L & $2.30(0.2)$ & $2.32(0.2)$ & 0.6 \\
\hline Serum phosphorus, $\mathrm{mmol} / \mathrm{L}$ & $1.96(0.5)$ & $2.01(0.8)$ & 0.8 \\
\hline Serum albumin, g/L & $38.1(3)$ & $38.0(3)$ & 0.9 \\
\hline PCR & $1.13(0.4)$ & $0.91(0.3)$ & 0.008 \\
\hline Treatment time, min & $232(20)$ & $234(17)$ & 0.7 \\
\hline Blood flow, $\mathrm{mL} / \mathrm{min}$ & $411(83)$ & $410(78)$ & 0.9 \\
\hline Dialysate flow, $\mathrm{mL} / \mathrm{min}$ & $674(106)$ & $683(121)$ & 0.8 \\
\hline
\end{tabular}

IBPSs, intradialytic blood pressure slopes; HD, hemodialysis; SBP, systolic blood pressure; DBP, diastolic blood pressure; TPRI, total peripheral resistance index; CI, cardiac index; ECW, extracellular water; TFC, thoracic fluid content; ET-1, endothelin 1; ADMA, asymmetric dimethylarginine; PTH, parathyroid hormone; PCR, protein catabolic rate. 


\section{Discussion}

The principal finding of this study is the quantification of the significant association between IBPS and the degree of ECV overload in hypertensive HD patients. The degree of ECV overload is more strongly associated with IBPS than it is with pre-HD, post-HD, or the overall change in systolic $\mathrm{BP}$ from pre to post-HD. The association between ECV overload and IBPS is preserved when adjusting for gender, diabetes, and ultrafiltration rate; it is strengthened in patients with post-hemodialysis BP in the hypertensive range. This study demonstrates the association between IBPS and a continuous, objective assessment of ECV and introduces a novel method to utilize routinely obtained clinical data to assist in making important clinical decisions in the HD unit.

Multiple observational studies in HD patients show that ECV overload increases mortality risk $[1,2,17,18]$. Improvements in diagnosing and managing ECV overload have been recently highlighted as a priority in the HD community [19]. Two recent clinical trials showed that integrating BIS measurements into fluid management decisions decreases ECV and may also reduce BP and mortality [20,21]. However, BIS is not part of routine clinical management. Patients with defibrillators or pacemakers cannot undergo these measurements for safety concerns, and the accuracy of BIS measurements is limited in patients with amputations or implanted metal. Finally, there are differences in the data provided by various BIS machines with some providing estimates of fluid overload, while others (including the only machines available in the United States) providing only the measured volume of the intracellular and extracellular spaces. Despite the accepted validity of BIS in general, there is no consensus on how this information should be interpreted or applied to clinical use. There remains a critical need for a method to assess ECV in the clinical setting using more readily and universally available data.

Peridialytic BP measurements provide some information on ECV, but the comparison of how ECV is associated with either individual BP measurements or repeated BP measurements has never been evaluated. Pre-HD BP measurements are strongly affected by interdialytic weight gain, a reflection of acute ECV expansion [22]. Conversely, post-HD BP better assesses chronic ECV overload [23]. Using post-HD ECW/body weight to define chronic ECV overload, our study confirmed that post-HD BP is a better surrogate of chronic ECV overload than pre-HD systolic BP. However, we showed that IBPS had a stronger correlation with chronic ECV overload than pre-HD, post-HD, or delta systolic BP did.

IBPSs and Volume Overload
The mean IBPS was negative in our study population, consistent with the typical intradialytic BP change in the general HD population [24]. Consistent with this finding, most of the multivariate analyses for IBPS demonstrated a negative intercept when controlling for the independent variables. Our findings demonstrate that for each unit increase in post-HD ECW/body weight, the IBPS becomes less negative, but for each unit decrease in post HD ECW/ body weight, the IBPS becomes more negative. When ECW/body weight was the dependent variable, the parameter estimate for IBPS remained positive, again showing that more positive slopes predicted greater ECV excess compared to more negative slopes. This is consistent with retrospective analysis of the DRIP trial, which found that subjects randomized to intense ultrafiltration for 8 weeks experienced steeper declines in intradialytic BP [25]. However, our study includes objective measurements of ECV using the BIS equipment to establish this relationship along a more continuous spectrum of volume states. Our findings also significantly expand on 3 recent case-control studies, which showed that patients with intradialytic BP increases had more volume overload based on BIS measurements than those with intradialytic BP decreases $[7,8,26]$. However, our study establishes that the repeated measurements included in the IBPS provide superior information than just the change in systolic $\mathrm{BP}$ from pre- to post-HD and that one can predict the relative degree of ECV overload based on the IBPS.

While IBPS had the strongest associations with postHD ECW/body weight, there were also significant associations with pre-HD ECW/body weight and post-HD thoracic fluid content and fluid overload. We feel the post-HD ECW/body weight is the best metric to assess chronic ECV overload because it reflects the fluid status after ultrafiltration has already occurred. Others have shown that BIS measurements in general remain stable for up to $2 \mathrm{~h}$ after a dialysis treatment [27]. Standardizing ECW based on body weight is an accepted metric for ECV excess and can be more sensitive at identifying ECV excess compared to ECW/TBW $[28,29]$. We appropriately controlled for gender, a factor known to influence ECW/ body weight, in our analyses. We consider thoracic fluid content and fluid overload to be of significant interest, but acknowledge these analyses were conducted for exploratory purposes.

We conducted a separate nonlinear sensitivity analysis of intradialytic BP. This approach also found a significant association between BP trajectory and the post-HD ECW/ body weight that was stronger than with any other ECV metric. Furthermore, this trajectory had a stronger asso- 
ciation with post-HD ECW/body weight than did pre$\mathrm{HD}$, post-HD, or delta systolic BP. Although the nonlinear approach accounts for the fact that most slopes were not perfectly linear, we believe that the linear model offers an advantage in being more clinically applicable due to easier calculation and interpretation. However, we ensured we were able to find thresholds where the linear model was most applicable. Including only those subjects with post-HD systolic BP $>130 \mathrm{~mm} \mathrm{Hg}$, the correlation strength increased to 0.40 . Prior epidemiologic research from large North American cohorts have identified that approximately $75 \%$ of HD patients have post-HD systolic BP higher than $130 \mathrm{~mm} \mathrm{Hg}[30,31]$ and nearly $90 \%$ have post-dialysis systolic BP measurements that exceed $120 \mathrm{~mm} \mathrm{Hg}$ [30]. The most updated KDOQI guidelines recommend targeting a post-HD systolic $\mathrm{BP}<130 \mathrm{~mm}$ $\mathrm{Hg}$ [11]. Thus, our model is most applicable to the majority of HD patients who require more intensive BP management. The associations between post-HD ECW/body weight and IBPS were weaker when considering only subjects with positive or negative slopes (though this was statistically significant in those with positive slopes in multivariate analysis). We interpret this to reflect both the reduction of power by reducing each sample size and the possibility that there may be a small effect difference with a stronger association in those with positive slopes. The improved correlation when considering the overall sample reinforces the fact that the IBPS metric can be useful in identifying covert ECV excess among a heterogeneous group of HD patients with different intradialytic BP patterns. Among subjects with more similar intradialytic BP patterns (i.e., a group of subjects who all have intradialytic hypertension), there are likely other discerning factors that determine the IBPS.

We suspect that the mechanism responsible for the association between IBPS and ECW/body weight is related to the persistent state of intravascular volume expansion in the context of ECV overload. It is known that higher ultrafiltration rates/volumes are associated with larger intradialytic decreases in BP [24]. While there is a strong negative correlation between ultrafiltration rate/volume and intravascular blood volume, there is a strong positive correlation between the post-HD volume overload and intravascular blood volume [32]. Importantly, the association between IBPS and ECW/body weight that we found was independent of the ultrafiltration rate. Diabetes was associated with IBPS in our study such that the presence of diabetes contributed to a more negative slope. This finding is likely related to the effects of diabetic neuropathy on autonomic nerve function. Still, the associa- tion between IBPS and ECV/body weight remained independent of the presence of diabetes. This was reinforced in separate subgroup correlation analyses of subjects with or without diabetes.

Because our previous study identified that increases in vascular resistance, and not cardiac output, were responsible for increase in BP in patients with intradialytic hypertension [8], it is possible that chronic intravascular volume expansion during hemodialysis induces a myogenic vasoconstrictive effect that maintains hemodynamic stability. We found in this study subjects with positive slopes were also more volume overloaded and had relative increases in TPRI compared to those with negative slopes. The association between TPRI and IBPS persisted even after controlling for age, ultrafiltration, and diabetes. We did not identify any association between IBPS and variables that would typically be considered to affect TPRI, including dialysate composition or changes in endothelial cell-derived vasomediators. In a prior crossover study of patients with intradialytic hypertension, dialysate sodium modification has been shown to change the course of intradialytic BP [33]. However, we had no direct measurements of plasma-dialysate sodium gradient or aldosterone to further assess the direct or indirect effects of sodium concentrations or gradients. We continue to find the combination of ECV overload and intradialytic TPRI rise to be of interest regarding how intravascular volume and intradialytic shifting of fluid into the intravascular space impact hemodynamics during the dialysis treatment.

Without any objective assessment of intravascular volume, such as relative blood volume monitoring (RBVM), it is not possible to confirm the possibility of a mechanistic association between intravascular volume and vasoconstriction. Other investigators have explored the relationship between RBVM and intradialytic BP. Sinha et al. [34] reported that the slope of the intradialytic hematocrit change assessed with RBVM was steeper in subjects who underwent intense ultrafiltration compared to controls in the DRIP trial. Furthermore, subjects with steep baseline slopes gained weight, but those with flat baseline slopes tolerated more fluid removal over time. So similar to IBPS, flat RBVM slopes are associated with ECV excess. The ability of one metric vs. the other to predict or guide the appropriate amount of ultrafiltration is unknown. A clinical trial testing the efficacy of RBVM in preventing intradialytic hypotension showed increased hospitalization and mortality in subjects assigned to RBVM [35], but the utility of prospectively assigning ultrafiltration based on retrospective analysis of the RBVM slopes has never been assessed. 
Limitations to this study include its moderate sample size and moderate correlation strength. A larger population would increase the likelihood of a correlation (decreased $p$ value) but not necessarily increase the strength of correlation ( $r$ value). We believe our subgroup analysis of various post-hemodialysis systolic BP cutoffs demonstrates that our method of ECV assessment is more applicable to some patients than others. A larger population would allow for more detailed subgroup analyses to better understand the patients for which this method is most appropriate. Another limitation is we do not demonstrate superiority of our method to BIS for achieving any outcome. We acknowledge BIS is validated to measure ECV in hemodialysis patients [5] but emphasize that it has several limitations, which accentuate the need for developing alternative methods. The prevalence of cardiovascular and peripheral vascular disease is extremely high in end-stage renal disease patients, and BIS cannot be used in many of these patients. The calculation of slopes, while not currently part of the standard clinical practice, would be much simpler to implement requiring only access to a computer.

In conclusion, increasing IBPS predicts a greater degree of chronic ECV overload based on the significant association between IBPS and the objective assessment of ECV using BIS. The specific ECV metric that has the strongest association with IBPS is post-HD ECW/body weight. IBPS are more strongly associated with ECV than other hemodialysis-unit BP metrics are, and these associations are strongest in patients with post-hemodialysis hypertension. Determination of IBPS is an innovative method to quantify the degree of ECV overload in hypertensive HD patients using easily obtained clinical data. Further application of this relationship in clinical trials could identify more objective means to prescribe ultrafiltration in this patient population.

\section{Acknowledgments}

This work would not be possible without the efforts of our research team nurses and coordinators: Cassie Livingston, Tiara Tyler, Tammy Lightfoot as well as the support of DaVita Dialysis company. The content is solely the responsibility of the authors and does not necessarily represent the official views of the National Institutes of Health (NIH). This date was presented as a poster at the American Society of Nephrology Annual Meeting 2017 in New Orleans, LA, USA.

\section{Ethics Statement}

All subjects signed written informed consent before any study procedures. The University of Texas Southwestern Medical Center Institutional Review Board approved the protocol. All procedures were in accordance of the Declaration of Helsinki. The study was part of a registered clinical trial (NCT01862497).

\section{Disclosure Statement}

The authors have no conflicts of interest to disclose. The primary results of this paper have not been published elsewhere. Some of the subjects included in this analysis were part of a previously published case-control study, although the present scope and analysis are novel work.

\section{Funding Source}

Support for this study comes from NIH 1K23DK09600701A1 Patient Oriented Career Development Award (PVB) and institutional support as the Dedman Family Scholar in Clinical Care (PVB). R.L. is supported by funding by the Cancer Prevention and Research Institute of Texas (RP150596). Research in this study was further supported by the National Center for Advancing Translational Sciences of the National Institute of Health under award number UL1TR001105 and the University of Texas Southwestern O'Brien Kidney Research Core (NIH grant P30DK079328).

\section{References}

1 Zoccali C, Moissl U, Chazot C, Mallamaci F, Tripepi G, Arkossy O, Wabel P, Stuard S: Chronic fluid overload and mortality in ESRD. J Am Soc Nephrol 2017;28:24912497.

2 Chazot C, Wabel P, Chamney P, Moissl U, Wieskotten S, Wizemann V: Importance of normohydration for the long-term survival of haemodialysis patients. Nephrol Dial Transplant 2012;27:2404-2410.

3 Agarwal R, Andersen MJ, Pratt JH: On the importance of pedal edema in hemodialysis pa- tients. Clin J Am Soc Nephrol 2008;3:153158.

4 Moon JR, Tobkin SE, Roberts MD, Dalbo VJ, Kerksick CM, Bemben MG, Cramer JT, Stout JR: Total body water estimations in healthy men and women using bioimpedance spectroscopy: a deuterium oxide comparison. Nutr Metab 2008;5:7.

5 Raimann JG, Zhu F, Wang J, Thijssen S, Kuhlmann MK, Kotanko P, Levin NW, Kaysen GA: Comparison of fluid volume estimates in chronic hemodialysis patients by bioimpedance, direct isotopic, and dilution methods. Kidney Int 2014;85:898908.

6 Voroneanu L, Cusai C, Hogas S, Ardeleanu S, Onofriescu M, Nistor I, Prisada O, Sascau R, Goldsmith D, Covic A: The relationship between chronic volume overload and elevated blood pressure in hemodialysis patients: use of bioimpedance provides a different perspective from echocardiography and biomarker methodologies. Int J Nephrol Urol 2010;42: 789-797. 
7 Nongnuch A, Campbell N, Stern E, El-Kateb S, Fuentes L, Davenport A: Increased postdialysis systolic blood pressure is associated with extracellular overhydration in hemodialysis outpatients. Kidney Int 2015;87:452457.

8 Van Buren PN, Zhou Y, Neyra JA, Xiao G, Vongpatanasin W, Inrig J, Toto R: Extracellular volume overload and increased vasoconstriction in patients with recurrent intradialytic hypertension. Kidney Blood Press Res 2016;41:802-814.

9 Park J, Rhee CM, Sim JJ, KIm YL, Ricks J, Streja E, Vashistha T, Tolouian R, Kovesdy CP, Kalantar-Zadeh K: A comparative effectiveness research study of the change in blood pressure during hemodialysis treatment and survival. Kidney Int 2013;84:795-802.

10 Van Buren PN, Kim C, Toto RD, Inrig JK: The prevalence of persistent intradialytic hypertension in a hemodialysis population with extended follow-up. Int J Artif Organs 2012;35: 1031-1038.

11 K/DOQI Workgroup: K/DOQI clinical practice guidelines for cardiovascular disease in dialysis patients. Am J Kidney Dis 2005;45(4 suppl 3):S1-S153.

12 ClinicalTrials.gov [Internet]. Bethesda, National Library of Medicine (US), 2000. With Intradialytic Hypertension. https:// www.clinicaltrials.gov/ct2/show/NCT01862 497?term=van+buren\&rank=2; NLM identifier NCT01862497 NIoDaDaKDNTUoTSMCaDMaToIABPiP (cited January 16, 2016).

13 Abbas SR, Zhu F, Levin NW: Bioimpedance can solve problems of fluid overload. J Ren Nutr 2015;25:234-237.

14 Abreo AP, Chertow GM, Dalrymple LS, Kaysen GA, Johansen KL: Association of bioimpedance spectroscopy-based volume estimation with postdialysis hypotension in patients receiving hemodialysis. Hemodial Int 2015;19:536-542.

15 Kossari N, Hufnagel G, Squara P: Bioreactance: a new tool for cardiac output and thoracic fluid content monitoring during hemodialysis. Hemodial Int 2009;13:512-517.

16 Engineer RS, Benoit JL, Hicks CW, Kolattukudy SJ, Burkhoff D, Peacock WF: Hemodynamic changes as a diagnostic tool in acute heart failure - a pilot study. Am J Emerg Med 2012;30:174-180.

17 Paniagua R, Ventura MD, Avila-Diaz M, Hinojosa-Heredia H, Mendez-Duran A, Cueto-
Manzano A, Cisneros A, Ramos A, MadoniaJuseino C, Belio-Caro F, Garcia-Contreras F, Trinidad-Ramos P, Vazquez R, Ilabaca B, Alcantara G, Amato D: NT-proBNP, fluid volume overload and dialysis modality are independent predictors of mortality in ESRD patients. Nephrol Dial Transplant 2010;25: 551-557.

18 Wizemann V, Wabel P, Chamney P, Zaluska W, Moissl U, Rode C, Malecka-Masalska T, Marcelli D: The mortality risk of overhydration in haemodialysis patients. Nephrol Dial Transplant 2009;24:1574-1579.

19 Weiner DE, Brunelli SM, Hunt A, Schiller B, Glassock R, Maddux FW, Johnson D, Parker $\mathrm{T}$, Nissenson A: Improving clinical outcomes among hemodialysis patients: a proposal for a "volume first" approach from the chief medical officers of US dialysis providers. Am J Kidney Dis 2014;64:685-695.

20 Hur E, Usta M, Toz H, Asci G, Wabel P, Kahvecioglu S, Kayikcioglu M, Demirci MS, Ozkahya M, Duman S, Ok E: Effect of fluid management guided by bioimpedance spectroscopy on cardiovascular parameters in hemodialysis patients: a randomized controlled trial. Am J Kidney Dis 2013;61:957965.

21 Onofriescu M, Hogas S, Voroneanu L, Apetrii M, Nistor I, Kanbay M, Covic AC: Bioimpedance-guided fluid management in maintenance hemodialysis: a pilot randomized controlled trial. Am J Kidney Dis 2014;64:111118.

22 Inrig JK, Patel UD, Gillespie BS, Hasselbad V, Himmelfarb J, Reddan D, Lindsay RM, Winchester JF, Stivelman J, Toto R, Szczech LA: Relationship between interdialytic weight gain and blood pressure among prevalent hemodialysis patients. Am J Kidney Dis 2007; 2007:108-118.e1-e4.

23 Leypoldt J, Cheung AK, Delmez JA, Gassman JJ, Levin NW, Lewis JA, Lewis JL, Rocco MV: Relationship between volume status and blood pressure during chronic hemodialysis. Kidney Int 2002;61:266-275.

24 Dinesh K, Kunaparaju S, Cape K, Flythe JE, Feldman HI, Brunelli SM: A model of systolic blood pressure during the course of dialysis and clinical factors associated with various blood pressure behaviors. Am J Kidney Dis 2011;58:794-803.

25 Agarwal R, Light RP: Intradialytic hypertension is a marker of volume excess. Nephrol Dial Transplant 2010;25:3355-3361.
26 Sebastian S, Filmalter C, Harvey J, Chothia MY: Intradialytic hypertension during chronic haemodialysis and subclinical fluid overload assessed by bioimpedance spectroscopy. Clin Kidney J 2016;9:636-643.

27 Di Iorio BR, Scalfi L, Terracciano V, Bellizzi $\mathrm{V}$ : A systematic evaluation of bioelectrical impedance measurement after hemodialysis session. Kidney Int 2004;65:2435-2440.

28 Davies SJ, Davenport A: The role of bioimpedance and biomarkers in helping to aid clinical decision-making of volume assessments in dialysis patients. Kidney Int 2014;86: 489-496.

29 van de Kerkhof J, Hermans M, Beerenhout C, Konings C, van der Sande FM, Kooman JP: Reference values for multifrequency bioimpedance analysis in dialysis patients. Blood Purif 2004;22:301-306.

30 Zager PG, Nikolic J, Brown RH, Campbell MA, Hunt WC, Peterson D, Van Stone J, Levey A, Meyer KB, Klag MJ, Johnson HK, Clark E, Sadler JH, Teredesai P: "U” curve association of blood pressure and mortality in hemodialysis patients. Medical Directors of Dialysis Clinic, Inc. Kidney Int 1998;54:561569.

31 Li Z, Lacson E Jr, Lowrie EG, Ofsthun NJ, Kuhlmann MK, Lazarus JM, Levin NW: The epidemiology of systolic blood pressure and death risk in hemodialysis patients. Am J Kidney Dis 2006;48:606-615.

32 Kron S, Schneditz D, Leimbach T, Aign S, Kron J: Vascular refilling is independent of volume overload in hemodialysis with moderate ultrafiltration requirements. Hemodial Int 2016;20:484-491.

33 Inrig JK, Molina C, D'Silva K, Kim C, Van Buren P, Allen JD, Toto R: Effect of low versus high dialysate sodium concentration on blood pressure and endothelial-derived vasoregulators during hemodialysis: a randomized crossover study. Am J Kidney Dis 2015;65: 464-473.

34 Sinha AD, Light RP, Agarwal R: Relative plasma volume monitoring during hemodialysis AIDS the assessment of dry weight. Hypertension 2010;55:305-311.

35 Reddan DN, Szczech LA, Hasselbad V, Lowrie EG, Lindsay RM, Himmelfarb J, Toto RD, Stivelman J, Winchester JF, Zillman LA, Califf RM, Owen WF Jr: Intradialytic blood volume monitoring in ambulatory hemodialysis patients: a randomized trial. J Am Soc Nephrol $2005 ; 16: 2162-2169$. 